\title{
PHOSPHORUS ANALYSIS OF PHOSPHORYLATED DURIAN SEED GUM BY WET AND DRY DIGESTION
}

\author{
N. F. Zebua ${ }^{1,4, *}$, E. D. L. Putra' ${ }^{1}$, U. Harahap ${ }^{2}$ and J. Kaban ${ }^{3}$ \\ ${ }^{1}$ Department of Pharmaceutical Chemistry, Universitas Sumatera Utara, Medan, Indonesia \\ ${ }^{2}$ Department of Pharmacology, Universitas Sumatera Utara, Medan, Indonesia \\ ${ }^{3}$ Department of Chemistry, Universitas Sumatera Utara, Medan, Indonesia \\ ${ }^{4}$ Department of Pharmacy, Universitas Tjut Nyak Dhien, Medan, Indonesia \\ *E-mail: nilsya.zebua@utnd.ac.id
}

\begin{abstract}
Phosphorus is a chemical element having a boiling point of $280^{\circ} \mathrm{C}$. One of phosphorus utilization is crosslinking phosphate on starch and gum. The research on the analysis of phosphorus level in phosphorylated starch mostly begins with dry digestion. Durian seeds contain gum which can be extracted with water and then phosphorylated with sodium trimetaphosphate (STMP). Therefore, this study aims to compare the results of phosphorus gum concentrate content of durian phosphorylated seed with dry and wet digestion method. Analysis of phosphorus content by wet digestionmethod at Gum Phosphate Durian (DGF) 1:1 and DGF 2:1, yielded $14.45 \%$ and $10.99 \%$. Phosphorus content with dry digestion method at DGF1:1 and DGF 2:1 gave result $0.69 \%$ and $0.32 \%$. The degree of substitution of DGF 1:1 and DGF 2:1 gave result 1.44, and 0.90. The surface morphology of DGF particles shows the fused surface with elements $\mathrm{C}, \mathrm{O}, \mathrm{Na}, \mathrm{K}$ and $\mathrm{P}$.
\end{abstract}

Keywords: Phosphorus, Crosslink, Analysis, Digestion, Durian Seed, Gum, STMP

(C) RASĀYAN. All rights reserved

\section{INTRODUCTION}

Phosphorus as a chemical element with the symbol $\mathrm{P}$ is so reactive that it is rarely found in nature in a single form. Phosphorus has an atomic number of 15 , atomic mass $30.9738 \mathrm{~g} / \mathrm{mol}$ with a phosphorus boiling point of $280.5^{\circ} \mathrm{C}^{1}$. The phosphorus element is generally bound to other chemical elements by forming various compounds or molecules such as phosphates. Disodium orthophosphate, trisodium orthophosphate, disodium diphosphate, sodium tripolyphosphate (STPP), sodium trimetaphosphate (STMP) are phosphate compounds used as food additives as emulsifiers. Crosslinking with STMP has the highest crosslinking degree at $\mathrm{pH}$ 8-9.5, STPP above $\mathrm{pH} 10$, while crosslinking with phosphate compounds other than STPP and STMP is less studied ${ }^{2}$. The compound with STMP cross-linked has the ability to swelling so it can be utilized in drug and food formulations. The phosphorylated guar gum compounds with STMP crosslinking agents will changes in physical and chemical properties after crosslinking ${ }^{3}$. In this study, STMP was used as crosslinking agents to maximize the utilization of durian seed gum.

Durian fruit produces a large amount of biomass waste which has the potential as a source of carbohydrates ${ }^{4}$. Durian seeds have a high carbohydrate content of starch and gum. Gum and starch have differences where gums include water-soluble polysaccharides while starches do not ${ }^{5}$. Durian seed gum is obtained by extracting the durian seed with water and then salting out with the addition of ethanol to obtain dry gum ${ }^{6,7}$. Research of phosphorylated durian seed gum with STMP has not been done so it is considered necessary to investigate the analysis of phosphorus levels and cross-link phosphate levels after cross-linked with STMP. Phosphorus analysis of phosphorus starch and sago starch phosphorylated was preceded by dry digestion method at $550^{\circ} \mathrm{C}$ and then determined by spechtrophotometry ${ }^{8,9}$. There is no study comparing the results of phosphorus level analysis with wet and dry digestion method of

Rasayan J. Chem., 11(4), 1572-1576(2018)

http://dx.doi.org/10.31788/RJC.2018.1144069

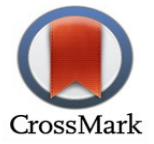


phosphorylated durian seed gum. The dry digestion uses a blast furnace with a temperature of $400-600^{\circ} \mathrm{C}$, so it is not relevant to analyze the phosphorus having a boiling point $280,5^{\circ} \mathrm{C}^{10}$. Based on the description of the nature of phosphor and the description of dry digestion and wet digestion it is necessary to analyze the comparison of phosphorus content by dry digestion method and wet digestion from the sample which has known phosphorus level is phosphorylated durian gum.

\section{Chemical and Equipment}

\section{EXPERIMENTAL}

The instruments used in this study are a set of UV-Vis spectrometer (Shimadzu), an infrared ray spectrophotometer (Shimadzu), scanning electron microscope-energy dispersive x-ray spectroscopy(Jeol 6510 LA), refrigerator (Toshiba), oven (Memmert), analytical balance (Sartorius), water purifier (Pure $\mathrm{Lab}$ ), hotplate stirrer (Boeco), magnetic bar, universal indicator (Merck), desiccator, $\mathrm{pH}$ meter, waterbath, and glassware. The materials used in this study were durian seed (Duriozibethinus Murr.) obtained from durian traders in Medan, ethanol (Merck), sodium trimetaphosphate (Merck), $\mathrm{HNO}_{3}$ (Merck), $\mathrm{H}_{2} \mathrm{SO}_{4}$ (Merck) Merck), potassium antimony loksitatrat, ascorbic acid, $\mathrm{NaOH}$ (Merck), and $\mathrm{KH}_{2} \mathrm{PO}_{4}$.

\section{Gum Extraction of Durian Seed}

$500 \mathrm{~g}$ of durian seed, peeled seeds, then mashed with a blender for $5 \mathrm{~min}$ with the addition of $5 \mathrm{~L}$ of distilled water, then added $2 \mathrm{M} \mathrm{NaOH}$ to $\mathrm{pH} 12$, then heated for $5 \mathrm{~min}$ and filtered. The supernatant placed in the refrigerator for $24 \mathrm{~h}$, the supernatant decanted from the residue and added $10 \mathrm{~L}$ of $96 \%$ ethanol, then placed in the refrigerator for $24 \mathrm{~h}$. The formed aggregate is filtered and washed with ethanol, then dried in a desiccator ${ }^{6,12}$.

\section{Gum Phosphate Synthesis}

$1 \mathrm{~g}$ of gum dissolved in $60 \mathrm{~mL}$ of water with hotplate stirred for $8 \mathrm{~h}$, and then $2 \mathrm{M} \mathrm{NaOH}$ added slowly to $\mathrm{pH}$ 8. The mixture is stirred until the solubilized is then added $1 \mathrm{~g}$ (DGF 1:1) or $500 \mathrm{mg}$ (DGF 2:1) of TNTMP which has been dissolved in $20 \mathrm{ml}$ of water.The film is dried in an oven at $40^{\circ} \mathrm{C}$ for $18 \mathrm{~h}^{3,11}$.

\section{Wet Digestion}

$1 \mathrm{~g}$ of film was added $1 \mathrm{~mL}$ of concentrated $\mathrm{HNO}_{3}$ and heated over a water bath. After cooling, 0,5 mL of $\mathrm{HNO}_{3} 5 \mathrm{~N}$ was added and redistilled until the solution became clear and the digestion finished with heating over the electric bath for $45 \mathrm{~m}^{10}$.

\section{Dry Digestion}

$5 \mathrm{~g}$ of film inserted into the porcelain, and then placed in a furnace at a temperature of $450^{\circ} \mathrm{C}$ for $24 \mathrm{~h}$. After cooling at room temperature, added $5 \mathrm{ml}$ of $\mathrm{HNO}_{3}$ and then placed back into the furnace. The ignition was carried out for $1 \mathrm{~h}$ and allowed to cool and placed to the desiccator ${ }^{8,9}$.

\section{Cross-Section Analysis of Cross-Phosphate Bonds}

The degree of substitution of phosphate from monophosphate and diphosphate durian seed gum is calculated by the formula ${ }^{8,14}$ :

DS $($ monophosphate $)=162 \mathrm{P} /(3100-103 \mathrm{P})$

DS $($ diphosphate $)=162 \mathrm{P} /(3100-102 \mathrm{P})$

Where $\mathrm{P}$ is calculated from the sample that was destructed by wet and dry digestion then $\mathrm{P}$ analysis with UV-Vis spectrophotometry, $162(180-18)$ is the molar mass of the anhydroglucose unit, 3100 is the mass of the phosphorus atom multiplied by 100,103 is thephosphate molar mass of the monophosphate, and 102 is the phosphate molar mass of diphosphate.

\section{RESULTS AND DISCUSSION}

Analysis of phosphorus content was done by using visible spectrophotometry method with wavelength obtained $722 \mathrm{~nm}$ and working time in the 45-51 minutes. The comparison of the results of the phosphorus level analysis by the method of wet digestion and dry digestion can be seen in Fig.-1. The degree of 
substitution monophosphate and diphosphate can be seen in Fig.-2. The particle surface morphology of the Durian Seed Gum Phosphorylated (DGF) shows the shape of the fused surface with elements C, O, $\mathrm{Na}, \mathrm{K}$ and $\mathrm{P}$ can be seen in Fig.-3.

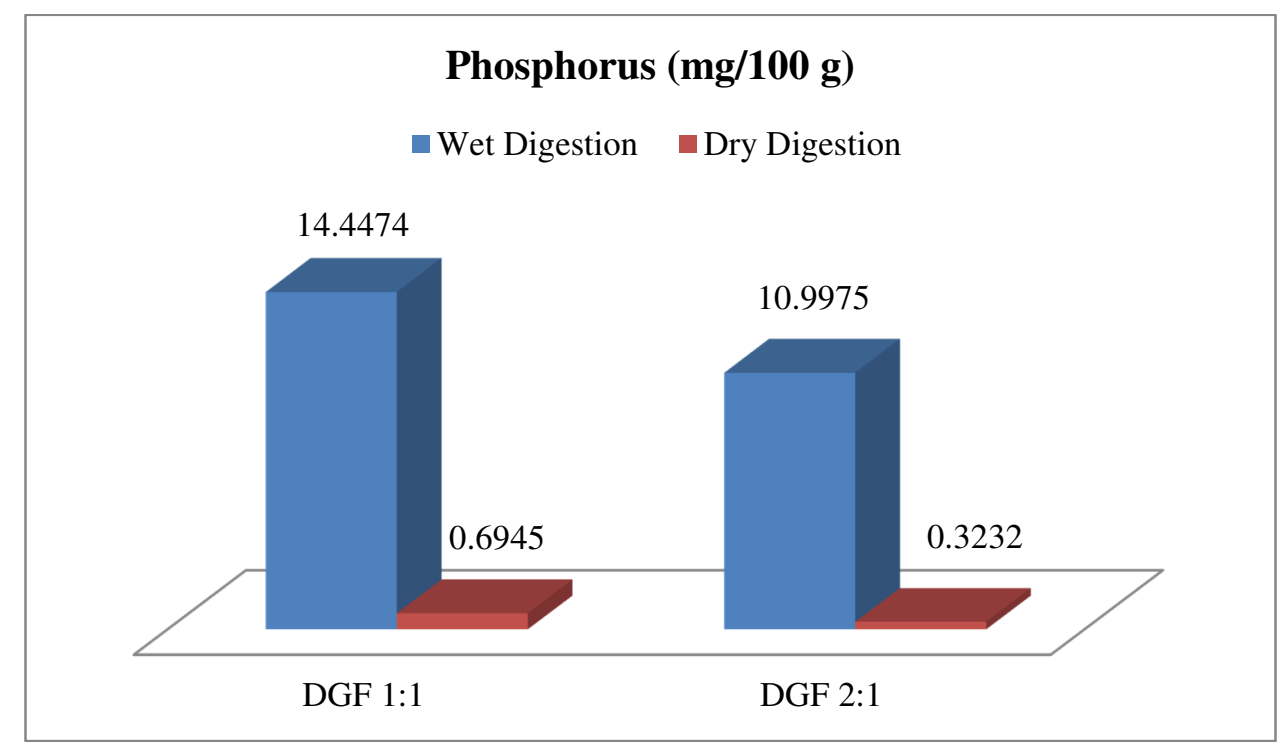

Fig.-1: Phosphorus Level Graph on Durian Seed Gum Phosphorylated (DGF) by Wet and Dry Digestion. Each value is the mean of six experiments.

DGF 1:1 is $1 \mathrm{~g}$ Durian Seed Gum with added $1 \mathrm{~g}$ STMP.

DGF 2:1 is $1 \mathrm{~g}$ Durian Seed Gum with added 0.5 g STMP

Figure -1 shows the results of analysis of phosphorus content obtained from durian seed gum and that has been phosphate bounded by two different methods of digestion. Levels of phosphorus in DGF 2:1 so lower than DGF 1:1 so it can be concluded that more STMP is added to the filmmaking process in line with increasing phosphorus bonded to gum, this conclusion needs to be proven by calculating the degree of crosslink phosphate. The morphology of particle surfaces before and after crosslinks with phosphates is noticeably different in Fig.-3. Durian seed gum still shows a hollow surface shape whereas phosphorylated gums look more fused, proving that the phosphate synthesis reaction in durian seed gum has occurred.This is in line with research on sago starch and rice starch that is after cross-linked with phosphate, the surface of starch particles will be more fused ${ }^{8}$.

The samples analyzed by dry digestion gave the result of very high cycling process and furnace temperature $\left(400^{\circ} \mathrm{C}\right)$ resulting in the number of missing samples in the process. The result of phosphorus analysis by wet digestion method and dry digestion showed a significant difference $(p<0.05)$. The result of wet digestion analysis is more acceptable because it shows the actual content that has been proven with recovery approaching $100 \%(96,32 \%)$ and relative standard deviation RSD <5\% $(1,43 \%)$.

The principle of dry digestion method is to evaporate carbon atoms, leaving the elements to be analyzed in the form of white ash. The dry digestion method is more commonly preferred in the metallic analysis because it does not use concentrated oxidizers so it is safer for the researcher but not all suitable elements are analyzed by this method. Elements that are destructed by dry digestion methods should have a boiling point higher than $550^{\circ} \mathrm{C}$ so as not to be lost in the analysis process.

Another method of digestion of an element to be known to the level is by the method of wet digestion. The phosphorus element has a strong enough bond to the carbon atom so that strong oxidizing agents are needed such as concentrated acids such as nitric acid, sulfuric acid, perchloric acid or all mixtures with heating aid ${ }^{1,10}$. The principle of this method is to break the organic bond into an ionic bond with a strong oxidant using a heating that is connected to a reflux set so that the metal having a low boiling point after boiling will be condensed and returned to its original container. This reflux allows perfect heating without worrying about the loss of the analyzed element. 
RASĀYAN J. Chem.

Vol. 11 | No. 4 |1572 - 1576| October - December | 2018

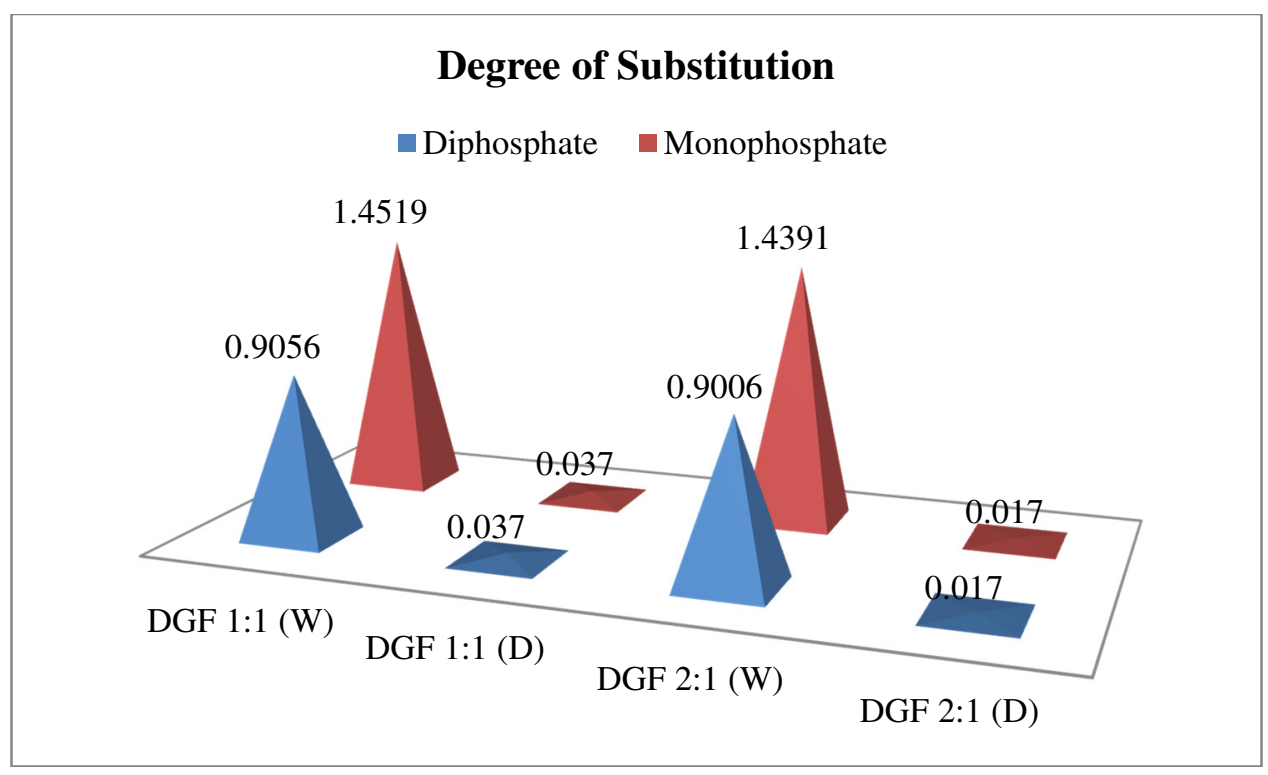

Fig.-2: Graph of Degree of Substitution Monophosphate and Diphosphate onDurian Seed Gum Phosphorylated. $\mathrm{W}$ is wet digestion, $\mathrm{D}$ is dry digestion.

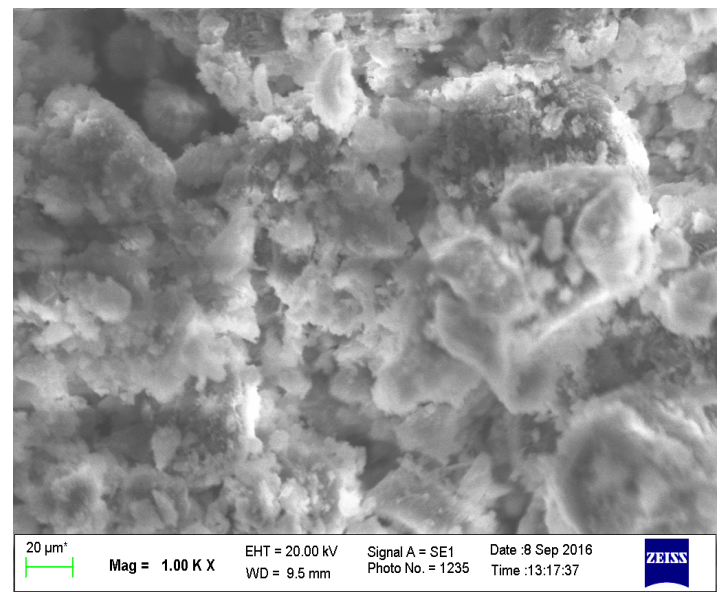

(a)

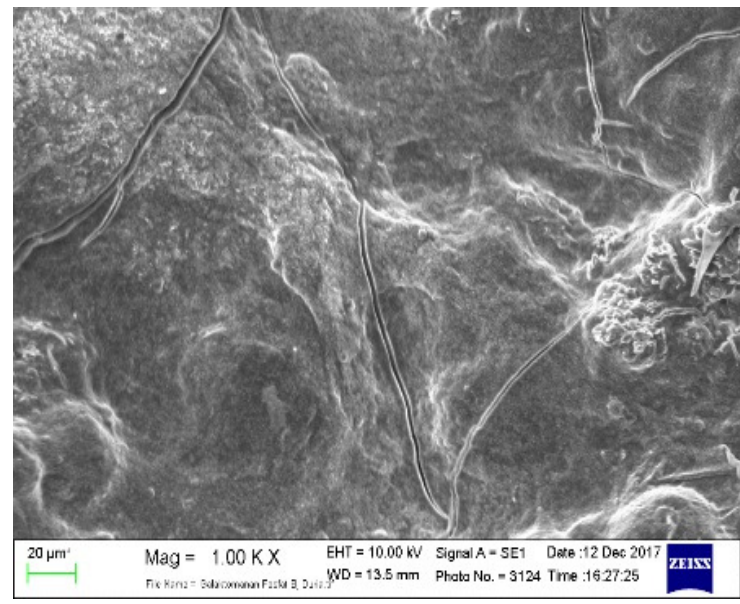

(b)

Fig.-3: The Particle Surface Morphology: (a) Durian Seed Gum and (b) Durian Seed Gum Phosphorylated (DGF 2:1)with 1000x magnification

The degree of crosslinking on rice starch can be analyzed by dry digestion method at $550^{\circ} \mathrm{C}^{7,8}$, but this does not apply to gum. Gum belongs to the oligosaccharide consisting of 2-6 monosaccharides, while the starch is classified as polysaccharides composed of more than 6 monosaccharides. The longer of $\mathrm{C}$ atoms in a compound will increase the boiling point of the compound. Phosphorus has atomic number 15, atomic mass $30.9738 \mathrm{~g} / \mathrm{mol}$ with phosphorus boiling point of $280.5^{\circ} \mathrm{C}$, so that at $400^{\circ} \mathrm{C}$ the phosphorus has evaporated so that the content becomes very small. The determination of total ash content is done at $450-600^{\circ} \mathrm{C}$ because at that temperature $\mathrm{C}$ atom is expected to have evaporated and left the remaining mineral or metal. Phosphorus analysis should use wet digestion using concentrated sulfuric acid, concentrated nitric acid or concentrated perchloric acid with heating less than $100^{\circ} \mathrm{C}^{11,13}$.

DGF 1:1 is a film of $1 \mathrm{~g}$ of durian seed and $1 \mathrm{~g}$ of STMP, while DGF 2:1 is a film of $1 \mathrm{~g}$ of durian seed and $500 \mathrm{mg}$ of STMP. The addition of STMP to DGF 2:1 did not show a doubling of phosphorus levels in DGF 1:1. The relative mass (RM) of phosphorus was 31 , while STMP molar mass $\left(\mathrm{Na}_{3} \mathrm{P}_{3} \mathrm{O}_{9}\right)$ was 306 , 
RASĀYAN $J$. Chem.

Vol. 11 | No. 4 |1572 - 1576| October - December | 2018

this means molar mass phosphorus 1/3 (30\%) of STMP molar mass, 15\% of DGF 1:1 and 10\% of DGF $2: 1$. This corresponds to the molar mass value of $\mathrm{P}$ is 93 which is $30 \%$ of STMP molar mass of 306.

\section{CONCLUSION}

The conclusion of this study is that the greater STMP added in the phosphate crosslinking process will further increase the degree of substitution of monophosphate and diphosphate and the best digestion method for analyzing phosphorus levels and the degree of phosphate substitution in durian seed gum is wet digestion.

\section{ACKNOWLEDGMENT}

Sincerely to the technological and higher education ministry that has supported this research through the Dissertation Research Scheme of 2018.

\section{REFERENCES}

1. K. S. Chrodter, G. Bettermann, T. Staffel, F. Wahl, T. Klein, T. Hoffmann, Wiley Online Library, DOI: 10.1002/14356007.a19_465.pub3

2. K. Muhammad, F.Hussin, Y.C.Man, H. M. Ghazali, J. F. Kennedy, Carbohydrate Polymer, 42, 85(1999), DOI:10.1016/S0144-8617(99)00120-4

3. I. G. Kabir, B. Yagen, A. Penhasi, A. Rubinstein, Journal of Controlled Release, 63, 121(2000).

4. M. Faisal, A. R.Yelvia Sunarti, H. Desvita, Rasayan J. Chem., 11(2), 871(2018). DOI:10.31788/RJC.2018.1123035

5. B. T. Amid, H. Mirhosseini, Food Chemistry, 132, 1258(2012), DOI:10.1016/j.foodchem.2011.11.099

6. N. F. Zebua, E. D. L. Putra, U. Harahap and J. Kaban, Asian Journal of Pharmaceutical and Clinical Research, 11, 174(2018), DOI:10.22159/ajpcr.2018.v11s1.26600

7. J. Kaban, J. Reveny, J. Tarigan, N. F. Zebua, Asian Journal of Pharmaceutical and Clinical Research, 11, 148(2018), DOI:10.22159/ajpcr.2018.v11s1.26593

8. F. J. Polnaya, Haryadi, D. W. Marseno, M. N. Cahyanto, International Food Research Journal, 20(4), 1609(2013)

9. P. Deetae, S. Shobsngob, W. Varanyanond, P. Chinachoti, O. Naivikul, S. Varavinit, Carbohydrate Polymers, 73, 351(2008), DOI:10.1016/j.carbpol.2014.07.028

10. M. Matek and M. Blanusa, Arhhig Rada Toksikol, 49(4), 301(1998).

11. J. Kaban, J. Reveny, J. Tarigan, N. F. Zebua, Rasayan J. Chem., 11(1), 294(2018), DOI: $10.7324 /$ RJC.2018.1112039

12. Nazliniwaty, Karsono, N. F. Zebua, Nerdy, Der Pharma Chemica., 8(20), 207(2016).

13. L. Edahwati, S. Sutiyono, S. Muryanto, J. Jamari, A. P. Bayuseno, Rasayan Journal of Chemistry, 11 (2), 904(2018), DOI:10.31788/RJC.2018.1123007

14. N. S. M. Sechi, P. T. Marques, Materials Research, 20(2), 174(2017), DOI:10.1590/1980-5373-MR2016-1008

[RJC-4069/2018] 